\title{
Scanning Electrochemical Microscopy as a Readout Tool for Protein Electrophoresis
}

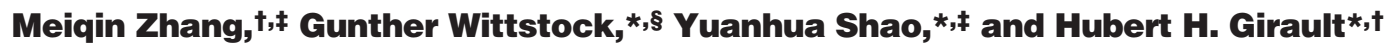 \\ Laboratoire d'Electrochimie Physique et Analytique, Ecole Polytechnique Fédérale de Lausanne, \\ CH-1015 Lausanne, Switzerland, Institute of Analytical Chemistry, College of Chemistry and Molecular Engineering, \\ Peking University, Beijing 100871, China, and Department of Pure and Applied Chemistry and Institute of Chemistry and \\ Biology of the Marine Environment, Faculty of Mathematics and Natural Sciences, Carl von Ossietzky University of \\ Oldenburg, D-26111 Oldenburg, Germany
}

Scanning electrochemical microscopy (SECM) was used to image silver-stained proteins on a poly(vinylidene difluoride) membrane. The method is based on measuring the current at a scanning microelectrode in the feedback mode. The electrochemical feedback is caused by the redox-mediated etching of the isolated 5-10-nm-diameter silver nanoparticles formed during the staining process. Several parameters, such as the redox mediator and the staining protocol, were optimized to ensure a high resolution and a low detection limit, i.e., $0.5 \mathrm{ng}$ of bovine serum albumin $\left(4 \times 10^{-14} \mathrm{~mol}\right)$ distributed on an area of $1 \mathrm{~mm}^{2}$ $\left(4 \times 10^{-16} \mathrm{~mol} \cdot \mathrm{cm}^{-2}\right)$. Images of $\beta$-lactoglobulin $A$ and myoglobin bands after gel electrophoretic separation and electroblotting were obtained in order to demonstrate that SECM can be employed as a sensitive and quantitative readout method for detection of proteins after gel electrophoresis. An additional advantage is that the silver staining can be removed, allowing further downstream mass spectrometry analysis.

Proteomics is the large-scale screening of the proteins of a cell, an organism, or a biological fluid, a procedure that includes sample preparation, 2-D electrophoresis, image detection and analysis, spot identification, and database searches. In parallel with the increasing demands to discover low-abundance proteins, the requirements for high sensitivity are continuously growing. ${ }^{1}$ Ideally, the gel electrophoresis approach should provide the lowest possible detection limit, a wide dynamic range, and a linear relationship between the amount of proteins present in the gel and the staining intensity. ${ }^{2}$ The detection procedure should be simple, fast to perform, nontoxic, mass spectrometry-compatible, and not too expensive from an application point of view. So far, various protein staining methods have been developed for visualizing proteins following gel electrophoresis, using visible organic

* Corresponding authors. E-mail: hubert.girault@epfl.ch. Tel: +41-21-693 3145. Fax: +41-21-693 3667. E-mail: yhshao@pku.edu.cn. Tel: +86-10-62759394. Fax: +86-10-62751708. E-mail: Gunther.Wittstock@Uni-Oldenburg.de. Tel: +49441-7983971. Fax: +49-441-7983979.

† Ecole Polytechnique Fédérale de Lausanne.

‡ Peking University.

$\$$ Carl von Ossietzky University of Oldenburg.

(1) Patton, W. F.; Beechem, J. M. Curr. Opin. Chem. Biol. 2001, 6, 63-69.

(2) Westermeier, R.; Marouga, R. Biosci. Rep. 2005, 25, 19-32. dyes, ${ }^{3,4}$ reverse staining with imidazole-zinc salts, ${ }^{5}$ copper staining, ${ }^{6}$ silver staining, ${ }^{7-15}$ fluorescence dyes staining, ${ }^{16,17}$ and radioactive labeling. ${ }^{18}$ Coomassie Brilliant Blue dyes have been very popular due to their low cost and relative ease of use. However, the major drawback of this method is its poor detection sensitivity, which is $\sim 8-50 \mathrm{ng}$ of protein. The fluorescence-based detection approach mainly suffers from the drawback of high cost due to the complex and expensive optical detection instrumentation. Although labeling of proteins using radioactive isotopes still remains the most sensitive method available, it requires special equipment and extremely complex handling procedures. For many years, silver staining with optical readout has been used as a relatively rapid, inexpensive, reproducible, and rather sensitive method. ${ }^{7-15}$ We propose here a further development of this approach by using a quantitative electrochemical readout method in order to determine the amount of silver associated with protein spots after electroblotting on a poly (vinylidene difluoride) (PVDF) membrane and silver staining.

Scanning electrochemical microscopy (SECM), a scanning probe technique, has been successfully developed into a powerful tool for studying heterogeneous and homogeneous reactions, for

(3) Fazekas de St Groth, S.; Webster, R. G.; Datyner, A. Biochim. Biophys. Acta 1963, 71, 377-391.

(4) Neuhoff, V.; Arold, N.; Taube, D.; Ehrhardt, W. Electrophoresis 1988, 9, 255-262.

(5) Fernandez-Patron, C.; Rodriguez, P.; Castellanos-Serra, L. Bio-Techniques 1992, 12, 564-573.

(6) Lee, C.; Levin, A.; Branton, D. Anal. Biochem. 1987, 166, 308-312.

(7) Switzer, R. C.; Merril, C. R.; Shifrin, S. Anal. Biochem. 1979, 98, 231-237.

(8) Oakely, B. R.; Kirsch, D. R.; Morris, N. R. Anal. Biochem. 1980, 105, 361363.

(9) Merril, C. R.; Dunau, M. L.; Goldman, D. Anal. Biochem. 1981, 110, 201207.

(10) Merril, C. R.; Pratt, M. E. Anal. Biochem. 1986, 156, 96-110.

(11) Blum, H.; Beier, H.; Gross, H. J. Electrophoresis 1987, 8, 93-99.

(12) Rabilloud, T.; Vuillard, L.; Gilly, C.; Lawrence, J. J. Cell. Mol. Biol. 1994, 40, 57-75.

(13) Shevchenco, A.; Wilm, M.; Vorm, O.; Mann, M. Anal. Chem. 1996, 68, $850-858$.

(14) Mortz, E.; Krogh, T. N.; Vorum, H.; Gorg, A. Proteomics 2001, 1, 13591363.

(15) Sørensen, B. K.; Højrup, P.; Østergård, E.; Jørgensen, C. S.; Enghild, J.; Ryder, L. R.; Houen, G. Anal. Biochem. 2002, 304, 33-41.

(16) Bermudez, A.; Daban, J.-R.; Garcia, J. R.; Mendez, E. Biotechniques 1994, 16, 621-624.

(17) Hart, C.; Schulenberg, B.; Steinberg, T. H.; Leung, W. Y.; Patton, W. F. Electrophoresis 2003, 24, 588-598.

(18) O’Farrell, P. H. J. Biol. Chem. 1975, 250, 4007-4021. 
high-resolution imaging of the chemical reactivity and topography of various interfaces and surfaces, and for microfabrication. ${ }^{19-22}$ Since the inception of SECM, it has been widely employed in studying biological systems or biomolecules immobilized onto various surfaces..$^{23-28}$ Instrumentally, SECM comprises a diskshaped ultramicroelectrode (UME) as a probe, a high-precision position controller, and a bipotentiostat. SECM possesses some unique features that cannot be rivaled by other scanning probe microscopic techniques (SPMs). For example, SECM is capable of imaging chemical or biochemical activities present at a surface and enables image frames with areas up to a few square centimeters. The latter feature makes SECM particularly advantageous over other SPMs for the imaging of proteins separated by gel electrophoresis (e.g., sodium dodecyl sulfate-polyacrylamide gel electrophoresis, SDS-PAGE) and then electroblotted on a PVDF membrane. Moreover, SECM is relatively simple, compact, and inexpensive compared with other SPMs and optical instrumentation. Finally, a well-developed theory of SECM facilitates quantitative protein detection.

The complex protein patterns obtained in 2-D gel electrophoresis need to be converted into a digital format for computeraided image analysis and data storage. At present, the image acquisition of the gel relies on camera systems or scanners with their inherent limitations in term of contrast and dynamic range. We had previously shown that the SECM approach could be used to detect silver- or copper-stained proteins..$^{29,30}$ These reports demonstrated the feasibility of using SECM for imaging protein spots on a surface. However, it was not yet shown that this principle can be applied to proteins after gel electrophoretic separation and electroblotting. In addition, the characteristic features of the SECM detection had not been investigated quantitatively.

The purpose of the present work is to improve the methodology and to demonstrate that SECM is a highly sensitive tool of imaging proteins separated by gel electrophoresis, electroblotted on PVDF membranes, and then silver stained. We show in particular that silver staining results in the formation of an array of unconnected metallic nanoparticles (5-10 $\mathrm{nm}$ in diameter). An interesting feature of the SECM readout is the possibility to fully remove the silver nanoparticles from the PVDF membrane in order for the proteins to be further analyzed by mass spectrometry.

(19) Scanning Electrochemical Microscopy; Bard, A. J., Mirkin, M. V., Eds.; Marcel Dekker: New York, 2001.

(20) Macpherson, J. V.; Slevin, C. J.; Unwin, P. R. J. Chem. Soc., Faraday Trans. 1996, 92, 3799-3805.

(21) Sun, P.; Li, F.; Chen, Y.; Zhang, M.; Zhang, Z.; Gao, Z.; Shao, Y. J. Am. Chem. Soc. 2003, 125, 9600-9601.

(22) Zhang, Z.; Yuan, Y.; Sun, P.; Su, B.; Guo, J.; Shao, Y.; Girault, H. H. J. Phys. Chem. B 2002, 106, 6713-6717.

(23) Horrocks, B. R.; Wittstock, G. in ref 13, pp 445-519.

(24) Pierce, D. T.; Unwin, P. R.; Bard, A. J. Anal. Chem. 1992, 64, 1795-1804.

(25) Wittstock, G.; Schuhmann, W. Anal. Chem. 1997, 69, 5059-5066.

(26) Liu, B.; Rotenberg, S. A.; Mirkin, M. V. Proc. Natl. Acad. Sci. U.S.A. 2000, 97, 9855-9860.

(27) Yasukawa, T.; Kaya, T.; Matsue, T. Electroanalysis 2000, 12, 653-659.

(28) Wittstock, G.; Burchardt, Malte.; Pust, S. E.; Shen, Y.; Zhao, C. Angew. Chem., Int. Ed. 2007, 46, 1584-1617.

(29) Carano, M.; Lion, N.; Abid, J.-P.; Girault, H. H. Electrochem. Commun. 2004, $6,1217-1221$.

(30) Carano, M.; Lion, N.; Girault, H. H. Chimia 2005, 59, 105-108.

\section{EXPERIMENTAL SECTION}

Chemicals and Materials. All chemicals were used as received: potassium hexachloroiridate(III) (Aldrich), potassium nitrate (>99\%, Fluka) tris(hydroxymethyl)aminomethane (Tris, 99.9\%, Sigma), SDS (99\%, Sigma), acrylamide/bis (30\% solution, Promega), glycerol anhydrous (>99.5\%, Fluka), bromophenol blue sodium salt (Fluka), ammonium persulfate (>98\%, Sigma), glycine (99\%, Sigma), $\beta$-mercaptoethanol (Bio-Rad, Hercules, CA), $N, N, N^{\prime}, N^{\prime}$ tetramethylethylenedlamine (TEMED; $99+\%$, Sigma), acetic acid (>99.8\%, Fluka), sodium acetate anhydrous (>99\%, Fluka), sodium thiosulfate pentahydrate (>99.5\%, Fluka), formaldehyde (37 wt \% solution in water, Aldrich), methanol (Merck), sodium carbonate anhydrous ( $>99.5 \%$, Fluka), silver nitrate (>99.5\%, Fluka), and sodium borohydride (power, 98\%, Acros). Immun-Blot PVDF membranes for protein blotting $(0.2 \mu \mathrm{m})$ were purchased from Bio-Rad and cut into circles to fit the electrochemical cell. Dual color (precision plus protein standards) was bought from BioRad. Osmium trisbipyridine chloride $\left[\mathrm{Os}(\mathrm{bpy}){ }_{3} \mathrm{Cl}_{2}\right.$ ] was kindly provided by Prof. Grätzel's group (Laboratory for Photonics and Interfaces, EPFL), and water was deionized using a model Milli-Q Plus 185 from Millipore (conductivity of $18.2 \mu \mathrm{S} \mathrm{cm}^{-1}$ ). Bovine serum albumin (BSA, $66.4 \mathrm{kDa}, \geq 98 \%$ ), myoglobin from horse heart $(16.7 \mathrm{kDa}, \geq 90 \%)$, and $\beta$-lactoglobulin A from bovine milk $(18.4 \mathrm{kDa}, \geq 90 \%)$ were purchased from Sigma. Alumina (1, 0.3, and $0.05 \mu \mathrm{m}$ ) and Mastertex polishing cloths from Buehler were employed to polish the UME.

Preparation of Protein Samples on PVDF Membranes. The hydrophobicity of PVDF makes it an ideal support for binding proteins. Because of the hydrophobic nature of PVDF, it was first wetted in methanol for $1-3 \mathrm{~s}$ and then immersed in water for 1-2 min to elute the methanol. (Caution: Once the membrane has been wetted with water, do not allow it to dry until the proteins have been transferred to it.) Proteins in pure water at some concentrations were spotted on the membranes by a microsyringe or by touching with a wetted razor blade. After evaporation of the solution, the membrane was stained with silver.

SDS-PAGE and Electroblotting to PVDF Membranes. SDSPAGE was performed essentially according to Laemmli in precast 10\% gels using Bio-Rad (Mini Protein II) ${ }^{31}$ The $10 \%$ resolving gels were mixed with $4 \mathrm{~mL}$ of $\mathrm{H}_{2} \mathrm{O}, 3.3 \mathrm{~mL}$ of $30 \%$ acrylamide mix, 2.5 $\mathrm{mL}$ of $1.5 \mathrm{M}$ Tris ( $\mathrm{pH} 8.8$ ), $0.1 \mathrm{~mL}$ of $10 \% \mathrm{SDS}, 0.1 \mathrm{~mL}$ of $10 \%$ ammonium persulfate, and $4 \mu \mathrm{L}$ of TEMED. The $5 \%$ stacking gels were mixed with $1.4 \mathrm{~mL}$ of $\mathrm{H}_{2} \mathrm{O}, 0.33 \mathrm{~mL}$ of $30 \%$ acrylamide mix, $0.25 \mathrm{~mL}$ of $1.0 \mathrm{M}$ Tris (pH 6.8), $20 \mu \mathrm{L}$ of $10 \%$ SDS, $20 \mu \mathrm{L}$ of $10 \%$ ammonium persulfate, and $2 \mu \mathrm{L}$ of TEMED. Samples were mixed with an equal volume of sample buffer, followed by loading of 20 $\mu \mathrm{L} /$ channel. Separations were performed for $60 \mathrm{~min}$ at $200 \mathrm{~V}$ and $60 \mathrm{~mA}$ in running buffer (25 mM Tris, $192 \mathrm{mM}$ glycine, 0.1\% SDS, $\mathrm{pH}$ 8.3).

After electrophoresis, gels were electroblotted to the PVDF membranes, which were prewetted by methanol using a Mini Trans-Blot Electrophoretic Transfer cell (Bio-Rad) for $1 \mathrm{~h}$ at a highintensity field (100 V, $350 \mathrm{~mA}$, 4-cm electrode distance). The blotting buffer was mixed with $25 \mathrm{mM}$ Tris and $192 \mathrm{mM}$ glycine in deionized water, $\mathrm{pH}$ 8.3. After transfer, the membranes were rinsed three times (5 min each) with distilled water prior to silver staining.

(31) Laemmli, U. K. Nature 1970, 227, 680-685. 


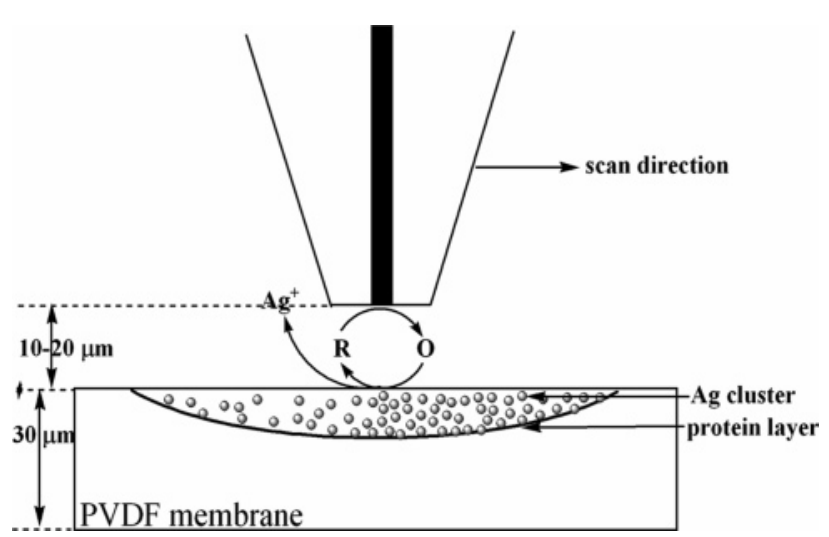

Figure 1. Schematic representation of SECM imaging of protein on the PVDF membrane by silver nanoparticle dissolution. Dimensions are not to scale.

Silver Staining of Proteins on PVDF Membranes. Two silver-staining protocols were tested and compared:

Protocol 1: This procedure is very similar to copper staining introduced by Lee et al. ${ }^{6} \mathrm{~A} 0.3 \mathrm{M} \mathrm{AgNO}_{3}$ solution was added to cover all the surface of the treated PVDF membrane, allowed to react with proteins for $\sim 30 \mathrm{~min}$, and then removed. After the sample was washed with copious amount of deionized water, a $0.3 \mathrm{M} \mathrm{NaBH}_{4}$ solution was added to reduce $\mathrm{Ag}^{+}$. After $\sim 5 \mathrm{~min}$, the solution was removed and the sample was carefully rinsed with deionized water. The sample is ready for the detection by SECM.

Protocol 2: This staining process was outlined by Sørensen et al. ${ }^{15}$ We only made a little modification after the sensitization step. The PVDF membrane was washed 3 times $5 \mathrm{~min}$ in 1\% sodium acetate. It was then rinsed twice with water for $1 \mathrm{~min}$. The membrane was sensitized 3 times $5 \mathrm{~min}$ in $0.1 \%$ sodium thiosulfate, and it was then rinsed with two changes of deionized water for 1 min. After rinsing, the membrane was submerged in chilled $0.1 \%$ silver nitrate solution (i.e., $6 \mathrm{mM}$ ) and incubated for $20 \mathrm{~min}$ at $4{ }^{\circ} \mathrm{C}$. After incubation, the silver nitrate was discarded, and the membrane was rinsed twice with water for $1 \mathrm{~min}$ and then developed in a mixture of $1 \%$ formalin and $2.5 \%$ sodium carbonate with intensive shaking for $10 \mathrm{~min}$. For double staining, this procedure was simply repeated. The development was stopped in $10 \%$ acetic acid for $5 \mathrm{~min}$. The membrane was dried for further characterization by optical laser-scanner (HP Scanjet 4890), SECM (CH Instruments model CHI 900), SEM (Quanta200FEG, FEI Co.), or TEM (Tecnai F30).

SECM Measurements. The experimental setup is schematically depicted in Figure 1. A three-electrode configuration was employed. The working electrode and scanning amperometric probe was a Pt UME prepared from a $20-\mu$ m-diameter Pt wire using the procedures described previously. ${ }^{32}$ A Laborlux D optical microscope (Leitz) is used to check the quality of the prepared UME. The UME is polished, washed with distilled water and acetone, and dried prior to each measurement. The RG value of the UME ( $R G=R_{\mathrm{g}} / r$, where $R_{\mathrm{g}}$ is the radius of the insulating glass) is between 3 and 4 . A Pt wire and a silver wire were used as the counter and quasi-reference electrodes, respectively. The PVDF membrane sample was sandwiched between a Teflon cell

(32) Lee, C.; Miller, C. J.; Bard, A. J. Anal. Chem. 1991, 63, 78-83. and a microscope glass slide. This cell was secured onto a platform. All of the SECM experiments were carried out at room temperature $\left(22 \pm 2{ }^{\circ} \mathrm{C}\right)$.

\section{RESULTS AND DISCUSSION}

Imaging of Protein Bands with Different Silver-Staining Protocols. BSA was chosen as a model protein. Immun-Blot PVDF membrane can bind peptides and proteins through hydrophobic interactions with a very high binding capacity (140-150 $\mu \mathrm{g} / \mathrm{cm}^{2}$ of membrane $\left.{ }^{33}\right)$. A protein band with very small size (width of $200-500 \mu \mathrm{m}$ ) was formed by touching the PVDF membrane with the edge of a razor blade that was wetted by the protein solution. After silver staining, the sample was mounted with the protein bands perpendicular to the $X$-axis of the SECM. Figure 1 shows the SECM imaging principle of silver-stained proteins on the PVDF membrane. In the amperometric feedback mode of SECM measurements, the UME signal used for imaging is a faradic current, $i$, originating from the oxidation of the mediator added to the electrolyte solution. The UME was kept at a constant potential $E_{\mathrm{T}}$ high enough to oxidize the chosen mediator dissolved in the bulk solution. Far above the sample surface, the anodic current $i$ is purely controlled by the hemispherical diffusion of the mediator toward the active disk of the UME and a diffusion-limited steady-state current $\left(i_{\infty}\right)$ is observed if the UME is held at a constant potential of $E_{\mathrm{T}}=+0.65 \mathrm{~V}$ versus the silver quasi-reference electrode in a $1 \mathrm{mM}\left[\mathrm{Os}(\mathrm{bpy})_{3}\right]_{\mathrm{Cl}_{2}}$ aqueous solution. When the UME is brought close to the silverstained protein sample, electrochemical recycling of the oxidizable mediator becomes possible by silver oxidation and mediator reduction. This leads to the increase of $i\left(i>i_{\infty}\right.$, positive feedback). On the other hand, $i$ will decrease as the UME approaches the insulating surface of the PVDF membrane without proteins $(i<$ $i_{\infty}$, negative feedback). For imaging of silver-stained proteins, UME approach curves $\left(i / i_{\infty}\right.$ versus UME-to-sample distance $d$ ) have been used to position the UME accurately at an appropriate $d$ over the PVDF sample. Changes in $i$ during lateral scans reflect gradually varying reaction rates for silver dissolution. The approach curves were recorded above an uncovered PVDF membrane using the mediator and evaluating the negative feedback. The approach was stopped when the UME mechanically touched the membrane. After approaching the membrane substrate, the UME was retracted $\sim 20 \mu \mathrm{m}$ and coarsely positioned close to the protein band for imaging.

Figure 2A shows SECM positive feedback behavior when the UME is scanned over a BSA band at a concentration of $10 \mathrm{mg} /$ $\mathrm{mL}$ stained with protocol 1 . The increase of $i$ is due to the reaction between the oxidized mediator $\left[\mathrm{Os}(\mathrm{bpy})_{3}\right]^{3+}$ and the silver clusters. Indeed, the standard redox potential of $\left[\mathrm{Os}(\mathrm{bpy})_{3}\right]^{3+/ 2+}$ equal to $0.834 \mathrm{~V}$ versus $\mathrm{SHE}$ is higher than that of $\mathrm{Ag}^{+} / \mathrm{Ag}$ equal to $0.8 \mathrm{~V}$ versus $\mathrm{SHE}$, or even less in the presence of chloride ions. When we scanned over the above area again, the current is lower (see Figure 2B), indicating that the silver is dissolved during the scanning process.

In order to compare the influence of silver-staining protocols on protein detection by SECM, two BSA bands (1 and $10 \mathrm{mg} /$ $\mathrm{mL}$ ) were imaged after silver-staining protocol 2 using the redox mediator $\left[\mathrm{IrCl}_{6}\right]^{3-}$ (standard redox potential, $0.867 \mathrm{~V}$ vs SHE). By

(33) Bio-Rad Laboratories, http://www.biorad.com, 2006. 

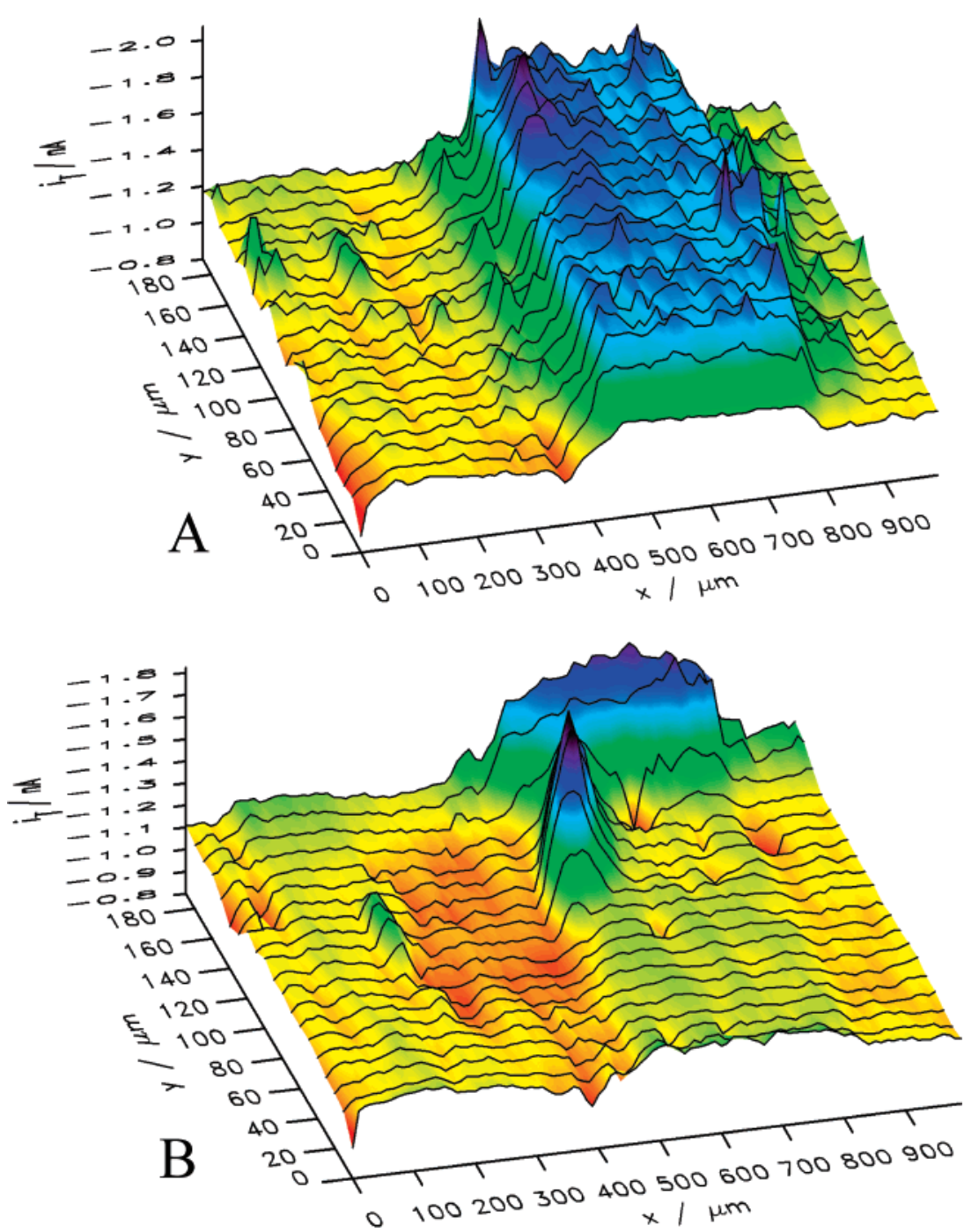

Figure 2. (A) SECM image of a $10 \mathrm{mg} / \mathrm{mL}$ BSA band with a $20-\mu \mathrm{m}$-diameter $\mathrm{Pt} U M E$. Measuring solution: $1 \mathrm{mM}\left[\mathrm{Os}(\mathrm{bpy})_{3}\right] \mathrm{Cl}_{2}$ in $0.1 \mathrm{M} \mathrm{KNO}$. $_{3}$ Scan speed: $50 \mu \mathrm{m} / \mathrm{s}$. (B) SECM image of the same area for $0 \leq y / \mu \mathrm{m}<180$ and unscanned area for $y / \mu \mathrm{m} \geq 180$.

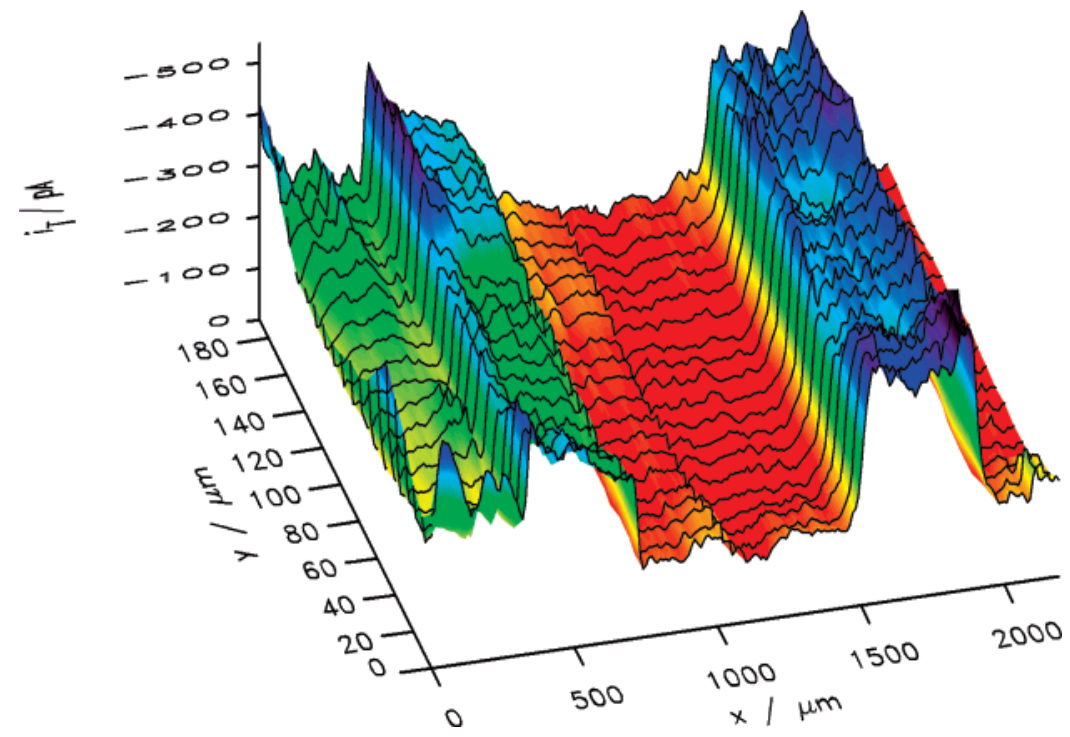

Figure 3. SECM image of two BSA bands at concentrations of 1 and $10 \mathrm{mg} / \mathrm{mL}$, respectively. The zone between $30<y / \mu \mathrm{m} \leq 130 \mathrm{has}$ been scanned over six times; the areas of $0 \leq y / \mu \mathrm{m} \leq 30$ and $130<y / \mu \mathrm{m} \leq 190$ have not been scanned before. Measuring solution: $1 \mathrm{mM} \mathrm{K}_{3}\left[\mathrm{IrCl}_{6}\right]$ in $0.1 \mathrm{M} \mathrm{KNO}_{3}$. Scan speed: $50 \mu \mathrm{m} / \mathrm{s}$. Probe: a $20-\mu \mathrm{m}$-diameter Pt UME.

adjustment of the staining conditions, it was possible to scan the same area several times without losing too much sensitivity.
Figure 3 shows an image of the two respective bands where the area has been scanned six times prior to recording this image. 
Only a signal loss of less than $20 \%$ is observed on the previously scanned area $(30<y / \mu \mathrm{m} \leq 130)$ compared with the regions that are imaged for the first time $(0 \leq y / \mu \mathrm{m} \leq 30$ and $130<y / \mu \mathrm{m} \leq$ 190).

As can be seen from Figures 2 and 3, both silver-staining protocols can be used for SECM detection of proteins, but protocol 2 allows repetitive and reproducible SECM imaging due to the relative small signal decrease after each image recording. In protocol 1 , the silver salt concentration used is large $(0.3 \mathrm{M})$ but the reducing time is relatively short ( $5 \mathrm{~min}$ ). It is likely to yield very small nanoparticles that can be easily redissolved as observed in Figure 2. In protocol 2, the silver salt concentration is much lower $(6 \mathrm{mM})$ but the reducing time is four times longer (20 min) and the resulting nanoparticles are much larger. In other words, protocol 1 favors the nanoparticle nucleation process but with a shorter growth period, whereas protocol 2 favors the formation of fewer nuclei that are allowed to grow to a larger size for a longer period.

If the nanoparticles were to coalesce to form a metallic silver film on the PVDF membrane, the oxidation of the mediator may also occur on the silver film away from the UME area, and no silver etching would be observed under the UME. Figures 2 and 3 therefore allow us to conclude that the silver-staining process generates isolated silver nanoparticles that are etched during the SECM readout. ${ }^{20}$ In order to verify this point further, we have analyzed the morphology of the silver particles generated by staining according to protocol 2 by electron microscopy (vide infra).

It should also be stressed that the present method of silver particle imaging differs from that proposed by Wang et al. for the imaging of DNA hybridization with $\mathrm{SECM}^{34}$ using gold nanoparticle-labeled oligodeoxynucleotide probes onto which silver had been deposited by electroless plating. In their case, the mediator $\left[\mathrm{Ru}\left(\mathrm{NH}_{3}\right)_{6}\right]^{3+}$ is reduced at the UME and reoxidized on the silverplated gold nanoparticles.

Characterization of PVDF Membranes and Silver Clusters by SEM and TEM. Since silver staining was first introduced in 1979 by Switzer et al., ${ }^{7}$ numerous silver-staining protocols have been described with different advantages regarding speed, sensitivity, cost, and compatibility with other analytical techniques, especially MS. ${ }^{7-15}$ However, the exact mechanism of silver staining in gel or PVDF membrane is still subject to debate, especially the protein-catalyzed nucleation process. Here, we have characterized the silver clusters made by protocol 2 by SEM and TEM. To this purpose, $5 \mu \mathrm{L}$ of a BSA solution ( $4 \mathrm{mg} / \mathrm{mL}$ ) was dropped on a PVDF membrane. This protein spot was then doubly silver stained as described above, and a brown spot with area of $\sim 1$ $\mathrm{mm}^{2}$ became visible on the membrane (not shown). Membrane fragments from this spot were further analyzed by SEM (Figure 4A) and TEM (Figure 4B). In Figure 4A, the silver nanoparticles appear as isolated particles on the membrane. With the aim to observe silver particles more clearly, a higher resolution image was obtained by TEM (Figure 4B). From these pictures, the diameters of the clusters on the PVDF membranes are estimated as $5-10 \mathrm{~nm}$.

Effect of Mediators on Sensitivity of Protein Detection. The effect of different mediators was investigated in order to

(34) Wang, J.; Song, F. Y.; Zhou, F. M. Langmuir 2002, 18, 6653-6658.
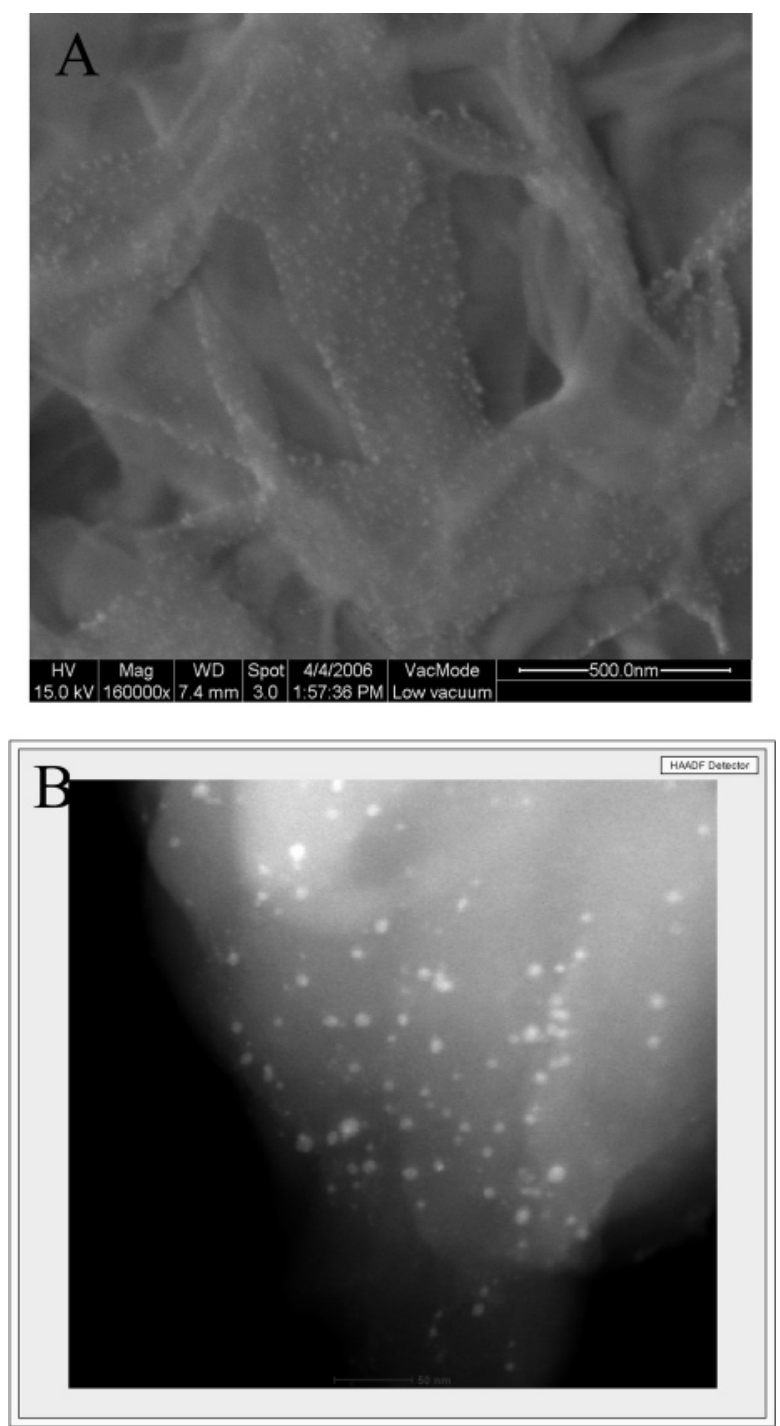

Figure 4. (A) SEM picture of silver clusters (inside the protein spot). (B) TEM picture of silver clusters inside the protein area.

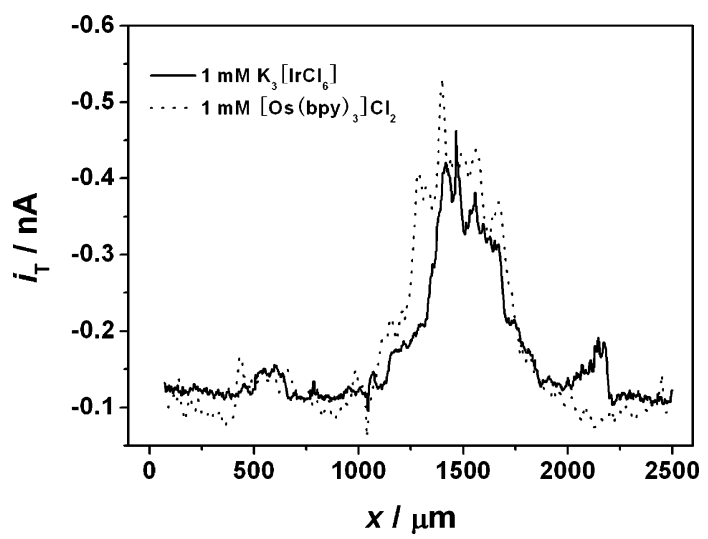

Figure 5. SECM image obtained by imaging two silver-stained BSA bands on the PVDF membrane at concentrations of 1 and $10 \mathrm{mg} / \mathrm{mL}$ with different mediators. Measuring solution: $1 \mathrm{mM} \mathrm{K}_{3}\left[\mathrm{IrCl}_{6}\right]$ in $0.1 \mathrm{M}$ $\mathrm{KNO}_{3}$ (solid line); $1 \mathrm{mM}\left[\mathrm{Os}(\mathrm{bpy})_{3}\right] \mathrm{Cl}_{2}$ in $0.1 \mathrm{M} \mathrm{KNO}_{3}$ (dotted line). All other conditions as in Figure 3.

achieve higher detection sensitivity. The sample was prepared with two BSA bands at concentrations of 1 and $10 \mathrm{mg} / \mathrm{mL}$ on the PVDF membrane and subsequently stained with silver according to 


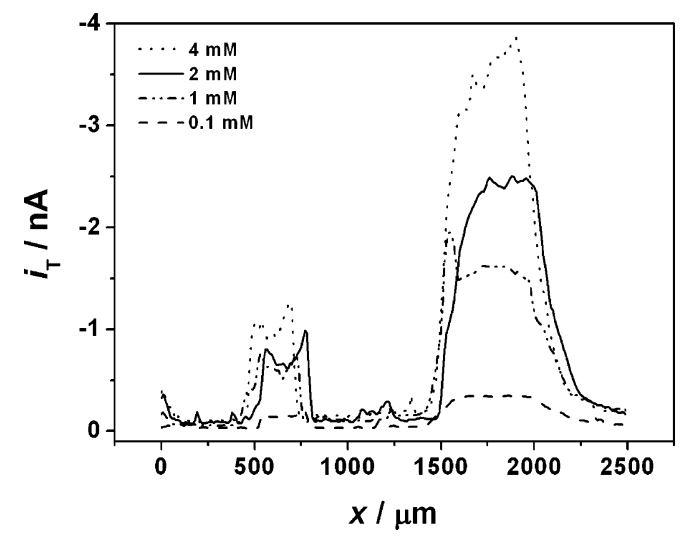

Figure 6. SECM image obtained by imaging two BSA bands at concentrations of 1 and $10 \mathrm{mg} / \mathrm{mL}$ with different $\mathrm{K}_{3}\left[|\mathrm{IrCl}|_{6}\right]$ concentrations: $0.16,1,2$, and $4 \mathrm{mM}$ in $0.1 \mathrm{M} \mathrm{KNO}_{3}$. All other conditions as in Figure 3.

protocol 2. Images were obtained by using both $\left[\mathrm{Os}(\mathrm{bpy})_{3}\right]^{2+}$ and $\left[\mathrm{IrCl}_{6}\right]^{3-}$ mediators. Figure 5 shows similar readouts for the highconcentration protein band. The low-concentration band is slightly better detected by $\left[\mathrm{IrCl}_{6}\right]^{3-}$. This may be due to the higher standard redox potential of $\left[\mathrm{IrCl}_{6}\right]^{3-/ 2-}$ compared to that of the osmium complex. In addition, due to the intense color of the osmium mediator solution, there are some difficulties with the coarse positioning of the SECM probe in the vicinity of the protein band on the PVDF membrane. Furthermore, the generated silver ion may react with the chloride in the solution to form a silver chloride precipitation when using $\mathrm{Os}(\mathrm{bpy})_{3} \mathrm{Cl}_{2}$ as the mediator, which also leads to the signal loss shown in Figure 2B. Therefore, this work shows that the commercial available $\mathrm{K}_{3}\left[\mathrm{IrCl}_{6}\right]$ is a more suitable mediator for routine protein imaging by SECM.

The effect of mediator concentrations on the detection level of protein by SECM was also examined. The sample was prepared in the same way as mentioned above. The SECM images over bands covered with different amounts of protein (Figure 6) show that the SECM feedback current increased monotonously with mediator concentrations changing from 0.16 to $4 \mathrm{mM}$. These data suggest that a $1 \mathrm{mM}$ concentration is sufficient to obtain high contrast images.

Detection Limit for Proteins on PVDF Membranes. The SECM image in Figure 7 shows parts of two protein spots of different concentrations. The samples were prepared by spotting drops with a microsyringe. The $0.5-\mu \mathrm{L}$ samples of 0.01 and 0.001 $\mathrm{mg} / \mathrm{mL} \mathrm{BSA}$ were spotted on the membrane, corresponding to final amounts 5 and $0.5 \mathrm{ng}$ of BSA, respectively. The spot areas are $\sim 1 \mathrm{~mm}^{2}$. After silver double staining according to protocol 2, the spots were imaged with $1 \mathrm{mM} \mathrm{K} \mathrm{K}_{3}\left[\mathrm{IrCl}_{6}\right]$. The minimum amount detected is $0.5 \mathrm{ng}$ of BSA $\left(4 \times 10^{-14} \mathrm{~mol}\right)$ distributed on an area of $\sim 1 \mathrm{~mm}^{2}\left(4 \times 10^{-16} \mathrm{~mol} / \mathrm{cm}^{2}\right)$ of the PVDF membrane. It should be noted that the spot of $0.5 \mathrm{ng}$ cannot be detected optically. The sensitivity depends of course not only on the readout but also on the silver-staining protocols. For instance, Sørensen et al. reported a silver-staining protocol of proteins on the PVDF membrane based on Gallyas' histochemical intensifier that reached a comparable sensitivity of the most sensitive silver-staining protocols for electrophoresis gels..$^{15} \mathrm{We}$ expect that, for any given silver-staining protocol, the SECM readout will have a detection limit lower than with optical camera systems or scanners.

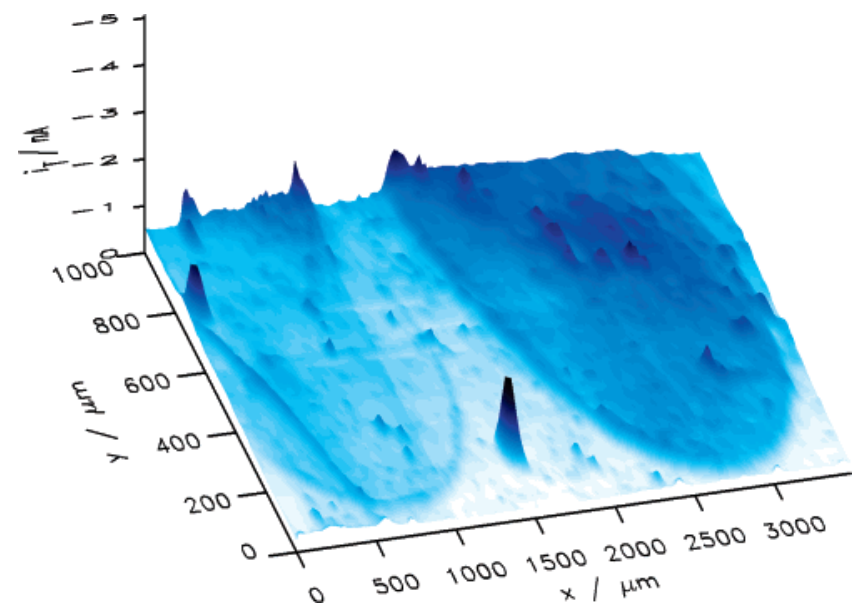

Figure 7. SECM image of a silver-stained sample with $0.5 \mu \mathrm{L}$ of BSA on the PVDF membrane at different concentrations: 0.01 (the right spot) and $0.001 \mathrm{mg} / \mathrm{mL}$ (the left spot). The size of protein spot is $\sim 1 \mathrm{~mm}^{2}$. The image frame covers a part of both spots. All other conditions as in Figure 3.

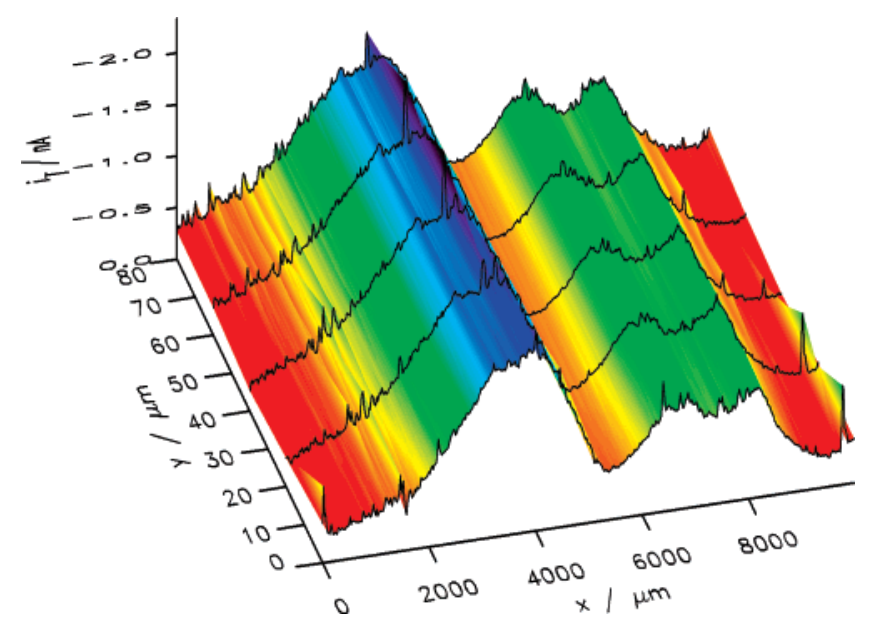

Figure 8. SECM image of proteins electroblotted from an electrophoresis gel including $\beta$-lactoglobulin A (left band) and myoglobin (right band). Sample: $10 \mu \mathrm{L}$ of $0.1 \mathrm{mg} / \mathrm{mL}$ of $\beta$-lactoglobulin A and $10 \mu \mathrm{L} 0.1$ of $\mathrm{mg} / \mathrm{mL}$ myoglobin for SDS-PAGE electrophoretic separation, electroblotting, and then silver staining (channel 8, Supporting Information). All other conditions as in Figure 3.

Imaging of Protein Bands after Electrophoresis. Having optimized several parameters (e.g., mediator compounds, staining protocol, mediator concentration) affecting the sensitivity of SECM imaging of stained protein spots on a PVDF membrane, we have carried out a SDS-PAGE electrophoresis and then electroblotted the SDS-denaturated proteins on a PVDF membrane. The transferred proteins were then silver stained on the membrane using protocol 2. At this point, it is unclear if the detergent SDS molecules were still present on the membrane when the staining process was performed. At that stage, the proteins appeared as brown bands on the substrate and an image was recorded using a laser-scanner (see Supporting Information). We then carried out a SECM image (Figure 8) of two closely spaced bands. The SECM image corresponds to a small portion of an electrophoresis channel where $\beta$-lactoglobulin A and myoglobin had been separated. The sample separated by SDS-PAGE electrophoresis contained $10 \mu \mathrm{L}$ of $0.1 \mathrm{mg} / \mathrm{mL} \beta$-lactoglobulin A and $10 \mu \mathrm{L}$ of 0.1 $\mathrm{mg} / \mathrm{mL}$ myoglobin. The SECM image was recorded with the high- 


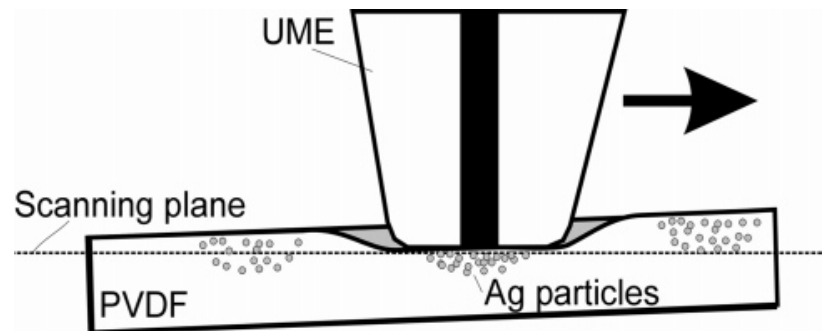

Figure 9. Schematic representation of the microelectrode indenting the flexible PVDF membrane and keeping a constant working distance to the $\mathrm{Ag}$ nanoparticles.

frequency axis perpendicular to the bands. The band of $2600 \leq$ $x / \mu \mathrm{m} \leq 5400$ is caused by $\beta$-lactoglobulin A that has a higher molecular weight than myoglobin detected at $6200 \leq x / \mu \mathrm{m} \leq$ 9000. The dimension of each band is about $9 \mathrm{~mm} \times 2.8 \mathrm{~mm}$, so the corresponding amount of protein is not higher than $40 \mathrm{ng} /$ $\mathrm{mm}^{2}$. Compared with optical readout by a laser-scanner of silverstained protein bands on the electroblotted PVDF membrane, a much clearer image of the two protein bands was acquired by SECM. This demonstrates the ability of SECM as a highly effective readout of electrophoretically separated and electroblotted proteins in low abundance. It also shows that traces of the chemicals used in a typical SDS-PAGE and electroblotting procedure do not significantly disturb the SECM detection.

Large-Area Scans and Current Enhancement. Somewhat to our surprise, we noted that we could perform line scans over several millimeters without experiencing extraordinary difficulties in preventing a tilt between the scanning plane and the sample surface. In all cases, the normalized current $i / i_{\infty}$ could reach values up to 6 corresponding to very small normalized distances. The experimental approach curves at an insulating area of a PVDF membrane and at an area covered with silver-stained proteins were recorded (see Supporting Information Figure 2). This is in agreement with our previous report (Figure 3 of ref 29) but in rather striking contrast to our experience from the work with hard samples. One possible explanation for the high normalized currents and the apparent insensitivity against sample tilt could be that the probe body indents (but does not penetrate!) the soft and porous PVDF membrane and slides on it without the typical stick-and-slip mechanisms (Figure 9). The low friction property of PVDF as a fluorinated polymer supports this possibility. By squeezing the porous PVDF membrane (Figure 4), the $\mathrm{Ag}$ nanopartticles are brought very close to the active UME area. In addition, mediator diffusion from the bulk solution is effectively blocked. Thus, the particular properties of the PVDF membranes can be exploited for the highly sensitive protein detection.

\section{CONCLUSIONS}

SECM in the feedback mode combined with silver staining has been proved to be a highly sensitive tool to image proteins separated by gel electrophoresis and subsequently blotted on the PVDF membrane. The minimum amount of protein detected is $0.5 \mathrm{ng} / \mathrm{mm}^{2}$ on the PVDF membrane. This result is already comparable to optimized staining techniques and optical detection used in membrane blotting analysis. Several parameters affecting the sensitivity of detection (e.g., type and concentration of mediator, staining protocol) were optimized. Meanwhile, SEM and TEM showed that the silver clusters in staining protocol 2 were isolated nanoparticles with $5-10$-nm diameters. This proves the proposed mechanisms as SECM-induced chemical etching of the isolated silver nanoclusters. A complete dissolution of the deposited silvers by the UME-generated mediator will facilitate protein recovery and downstream analysis, typically performed by MS. Work is in progress to reduce the gel size in order to make it more compatible with the SECM length scale and to cover a larger part of an entire electrophoresis graph in one image.

\section{ACKNOWLEDGMENT}

G.W. is grateful to the EPFL for a visiting professorship, and M.Z. is grateful to the NSFC for research grants (20235010 and 20420130137) and to the EPFL for a visiting postgraduate fellowship. We also thank Dr. B. Su, M. Abonnenc, and H.-T. Lam for their help during the SDS-PAGE and electroblotting experiments and Dr. H. Hu for SEM measurement. The technical assistance by Valérie Devaud is also acknowledged.

\section{SUPPORTING INFORMATION AVAILABLE}

Additional information as noted in text. This material is available free of charge via the Internet at http://pubs.acs.org.

Received for review December 13, 2006. Accepted April 23, 2007.

AC062356K 